\title{
Les enseignants et le dictionnaire : sentiments, attitudes motivationnelles, connaissances déclarées et pratiques personnelles d'utilisation
}

Ophélie Tremblay

Université du Québec à Montréal

Isabelle Plante

Université du Québec à Montréal

Catherine Fréchette-Simard

Université du Québec à Montréal
Paper and electronic dictionary use by
elementary and
secondary teachers in Quebec:
attitudes, knowledge and practices

doi:10.18162/fp.2018.452

\section{ésumé}

Le présent article documente le type de sentiments et les attitudes motivationnelles de 300 enseignants québécois du primaire et du secondaire face aux dictionnaires papier et électroniques, ainsi que leurs connaissances dictionnairiques et pratiques d'utilisation déclarées. Les résultats révèlent que les enseignants entretiennent des sentiments positifs envers les dictionnaires. De plus, ils se sentent généralement compétents dans l'utilisation de cet ouvrage et y accordent un degré de valeur élevé,

en plus de détenir des ouvrages variés. Cependant,

les connaissances et usages déclarés demeurent relativement rudimentaires, ce qui souligne les besoins de formation pour une meilleure exploitation personnelle et didactique des dictionnaires.

Mots-clés

Enseignants (primaire et secondaire), dictionnaire papier, dictionnaire électronique, attitudes motivationnelles, connaissances et pratiques déclarées.

Abstract

This article documents, among 300 elementary and high school teachers, the type of emotions, motivational attitudes, knowledge and personal uses of paper and electronic dictionaries. Results reveal that overall teachers have positive emotions towards paper and electronic dictionaries. Teachers also generally feel competent to use the dictionary and attribute a positive value to this tool. In addition, teachers from all grade levels own various dictionaries. Nevertheless, dictionary

knowledge and uses remain relatively basic, thus underlying the need for a better training about paper and electronic dictionaries in order to enhance teachers' personal and pedagogical dictionary use.

Keywords

Teachers (elementary school and high school), paper dictionary, electronic dictionary, attitudes, dictionary knowledge and use.

\section{Introduction}

Le dictionnaire, un gros volume poussiéreux dans lequel les mots suivent sagement l'ordre alphabétique? Il serait plus juste d'y référer comme étant un ouvrage riche, multidimensionnel (notamment dans sa version électronique), fruit d'un travail de conception rigoureux et approfondi, rempli d'informations précieuses pour mieux comprendre et utiliser les mots de la langue, moyennant un certain nombre de connaissances et d'habiletés dites " dictionnairiques ».

Enseigner à consulter un dictionnaire, papier ou électronique, fait d'ailleurs partie des prescriptions ministérielles au Québec, comme le précisent les programmes de formation. Ainsi, dès le premier cycle du primaire, l'enseignant devrait amener l'élève à se familiariser avec cet outil, en lui faisant observer différents types de dictionnaires imprimés et électroniques: imagiers, dictionnaires thématiques, descriptifs, des synonymes, etc. (Ministère de l'Éducation, du Loisir et du Sport [MELS], 2011a). Au fil de sa scolarité primaire, lélève devrait également apprendre à reconnaitre les principales abréviations utilisées dans un article de dictionnaire, de même que le type d'éléments qui structurent une entrée : classe de mots, genre des noms, registre, définitions correspondant aux divers sens d'un mot. Au secondaire, il aura à recourir aux dictionnaires papier et électroniques pour repérer les sens (propres et figurés) d'un mot, son orthographe, son origine, etc. (MELS, 2011b). Lélève apprendra aussi à se servir d'une plus grande variété de ressources en fonction de ses besoins de scripteur ou de lecteur : dictionnaire des champs lexicaux, dictionnaire de cooccurrences, dictionnaires des anglicismes, etc. Enfin, au deuxième cycle du secondaire, l'article de dictionnaire sera étudié comme un genre textuel, à dominante descriptive, doté de ses propres caractéristiques communicationnelles, textuelles, graphiques et visuelles (Chartrand et Émery-Bruneau, 2013). 
Ces prescriptions ministérielles mettent en lumière deux ingrédients essentiels pour une consultation efficace du dictionnaire, quel que soit son format ou son support : 1) un dictionnaire de qualité, facile à utiliser, et 2) un usager qui sait comment l'utiliser (Lew, 2013). Par conséquent, afin d'enseigner adéquatement aux élèves le maniement de cette ressource, les enseignants du primaire et du secondaire doivent, d'une part, disposer pour eux-mêmes (et en classe) de dictionnaires de qualité, en plus de maitriser les connaissances et habiletés nécessaires à une consultation efficace de ces ouvrages, aussi bien dans leur format papier qu'électronique. Or, peu de recherches encore attestent de la capacité des enseignants à utiliser le dictionnaire et, parallèlement, à en enseigner l'utilisation à leurs élèves, notamment dans un contexte francophone. De plus, une piste peu explorée concerne les attitudes motivationnelles des enseignants face à cet outil, des variables qui pourraient constituer un facteur déterminant de l'usage du dictionnaire en classe. En effet, il y a lieu de penser que les enseignants qui manifestent des attitudes motivationnelles positives envers le dictionnaire, se traduisant par un état émotionnel positif ainsi que par un sentiment de compétence et l'attribution d'un degré de valeur élevés face au dictionnaire (voir par exemple Eccles et Wigfield, 2002), y recourront fréquemment en classe avec leurs élèves. Si quelques études ont mis en évidence les conceptions limitées des enseignants et leurs connaissances lacunaires quant au contenu et aux usages du dictionnaire, ainsi qu'à la diversité et aux caractéristiques des ouvrages disponibles sur le marché (Anctil, 2010; Carstens, 1995; Neubach et Cohen, 1988; Scholfield, 1982), la question de leurs attitudes motivationnelles demeure inexplorée.

Quelle est la situation actuelle au Québec? Notre étude entendait répondre à cette question, en dressant un portrait des pratiques, connaissances, sentiments et attitudes motivationnelles déclarés par les enseignants en ce qui a trait aux dictionnaires électroniques et papier et à leurs usages. Une meilleure connaissance de ces éléments nous parait nécessaire afin d'identifier les lacunes potentielles dans les pratiques relatives à l'utilisation du dictionnaire par les enseignants. Sur la base de ces connaissances nouvelles, il sera possible de mieux déterminer les contenus de formation initiale et continue à offrir. Ce faisant, les élèves pourront recevoir un enseignement plus adéquat, pour tirer profit au maximum des fonctionnalités offertes par les dictionnaires, tant papier quélectroniques, afin de développer leurs compétences en lecture et en écriture.

\section{Les enseignants et le dictionnaire : quelques aspects théoriques}

\section{Le rôle des attitudes motivationnelles et des sentiments dans l'enseignement d'une discipline}

Les attitudes motivationnelles sont un important déterminant des comportements des individus (Bandura, 1977; Eccles et Wigfield, 2002). En contexte scolaire, l'importance de diverses attitudes motivationnelles des élèves (incluant la perception de compétence, la valeur ou l'intérêt accordé à un objet d'apprentissage) dans les comportements scolaires - tels l'effort, l'engagement ou le rendement - a été bien démontrée (pour des revues des écrits, voir Covington, 2000; Linnenbrink-Garcia, Tyson et Patall, 2008). Parallèlement, l'aspect affectif ou émotionnel est également réputé jouer un rôle prépondérant dans la mise en œuvre de comportements scolaires positifs (par exemple, Baumeister et Bushman, 2007; Duchesne, Vitaro, Larose et Tremblay, 2008). À son expression la plus simple, l'état émotionnel peut se réduire à un état positif, caractérisé par des sentiments positifs tels la joie, le plaisir ou la satisfaction, d'une part, ou négatif, se traduisant par des sentiments négatifs tels l'anxiété, le mécontentement ou l'ennui, d'autre part (Denham, 1998; Pekrun, Elliot, et Maier, 2009). 
Plusieurs études ont déjà montré la pertinence des attitudes motivationnelles chez les adultes pour comprendre leurs comportements en contexte de travail (par exemple, Gagné et Deci, 2009) et en particulier dans les pratiques pédagogiques d'enseignants. Ainsi, les enseignants qui entretiennent des perceptions positives relativement à leurs capacités pédagogiques, en combinaison avec un affect positif, développent une plus grande satisfaction professionnelle (Moè, Pazzaglia et Ronconi, 2010). Les attitudes motivationnelles contribuent également à la modification des pratiques enseignantes vers l'adoption de pratiques plus efficaces (Holzberger, Philipp et Kunter, 2013). Or, les attitudes motivationnelles et les sentiments des enseignants spécialement en ce qui a trait au dictionnaire nont, à notre connaissance, jamais été documentés, ce que la présente étude entendait faire.

\section{Les connaissances et habiletés à la base d'un processus de consultation réussi}

La consultation du dictionnaire fait appel à un ensemble de connaissances théoriques et d'habiletés pratiques (Fuertes-Olivera et Tarp, 2011; Lew, 2013; Scholfield, 1999), qui interviennent aussi bien avant le processus de consultation lui-même que durant ce dernier (Nesi, 1999), par exemple : 1) connaitre les types de dictionnaires et ce qu'ils contiennent puis choisir quel dictionnaire répond au besoin de consultation; 2) décider quel mot chercher et localiser l'entrée recherchée; 3) comprendre la structure du dictionnaire et l'ordre alphabétique, comprendre la correspondance entre le son et les lettres; 4) distinguer les différentes parties de l'entrée et les informations pertinentes, trouver l'information sur l'orthographe, le sens, l'étymologie, la morphologie, la syntaxe, la prononciation du mot, les exemples, les collocations, les mots dérivés, etc.

Dans la présente étude, deux éléments de cette liste ont été plus particulièrement ciblés, parce qu'ils correspondent à des points identifiés dans les programmes ministériels. Le premier concerne la connaissance des types de dictionnaires : les dictionnaires sur le marché ont des caractéristiques qui leur sont propres et qui détermineront le choix d'un usager averti en contexte de lecture ou d'écriture. Ces dictionnaires (dictionnaires bilingues, étymologiques, spécialisés ou dictionnaires de langue), qu'ils se présentent en format papier ou électronique, répondent en effet à des besoins distincts : traduire un mot ou en chercher l'équivalent dans une autre langue, retracer l'origine d'un mot, connaitre la définition d'un terme technique, ou simplement rechercher une information lexicale dans un article de dictionnaire courant. En ce qui a trait aux dictionnaires de langue en particulier, chacun possède des caractéristiques propres, qui répondront de façon différenciée aux besoins du scripteur. Par exemple, le Multidictionnaire sera indispensable pour corriger l'orthographe et la grammaire d'un texte, en plus de cibler les mots familiers et les anglicismes à éviter. Pour enrichir le vocabulaire d'un texte, il vaudra mieux recourir au Petit Robert ou encore à Antidote, qui proposent des descriptions fines et détaillées du sens des mots, des termes qui leur sont sémantiquement rattachés et des contextes d'utilisation variés dans lesquels ils apparaissent, en plus de fournir des indications sur l'étymologie et l'histoire des mots. Dans un contexte de classe où les élèves n'auraient pas accès à Internet, le Petit Larousse, dont la vocation est davantage encyclopédique, pourrait être utile à la rédaction d'un texte informatif, par exemple, grâce aux planches thématiques illustrées qu'il propose et aux nombreuses illustrations qu'il contient. À cet égard, une étude menée par Bernier (2010) a montré que des étudiants du collégial ayant suivi une formation sur diverses ressources électroniques comme Antidote, le Multidictionnaire de la langue française et le Petit Robert ont ensuite consulté davantage de ressources afin de résoudre diverses difficultés en écriture. 
En outre, la connaissance des ouvrages existants, de leurs caractéristiques et des besoins auxquels ils peuvent répondre est de plus en plus nécessaire dans un contexte numérique, où la grande offre de ressources lexicales disponibles sur le Web rend plus difficile la tâche de séparer les ressources de qualité de celles qui contiennent des informations moins précises ou moins détaillées (Lew, 2013). Nous avons donc émis l'hypothèse qu'un enseignant qui dispose pour lui-même d'une variété de dictionnaires sera mieux à même de connaitre et d'enseigner les caractéristiques de ces ouvrages et les contextes dans lesquels y recourir.

Le deuxième élément que la présente étude a ciblé concerne la connaissance des différentes composantes d'un article de dictionnaire. La figure 1 ci-dessous, tirée du site "Ouvrir le dictionnaire ", illustre ces composantes à l'intérieur de deux dictionnaires : le Multidictionnaire de la langue française (édition de 2009), à gauche, et Le Petit Robert (édition de 2012), à droite. On trouve dans cet extrait les informations suivantes : la classe du mot décrit, ses différents sens, sa prononciation en alphabet phonétique entre crochets (dans Le Petit Robert seulement), l'étymologie (dans Le Petit Robert seulement), les renvois vers des mots dérivés sémantiquement (les petites flèches noires dans Le Petit Robert), les formes fautives (après les astérisques dans le Multidictionnaire) et d'autres informations encore.

\begin{tabular}{|c|c|}
\hline $\begin{array}{l}\text { SPÉCIAL, IALE, IAUX adj. } \\
\text { 1. Qui s'applique exclusivement à une personne, à une } \\
\text { chose. Un visage très spécial. SYN. caractéristique; propre à; } \\
\text { singulier. ANT. commun. } \\
\text { - Ne pas confondre avec ladjectif spécifique, qui est carac- } \\
\text { téristique d'une espèce. } \\
\text { 2. Particulier, destiné à une personne, à un groupe. Une } \\
\text { classe spéciale de rattrapage. Des timbres spéciaux. Achetez } \\
\text { l'édition spécialel sYN. exceptionnel. ANT. courant. } \\
\text { 3. Bizarre, non courant. Ce vêtement est un peu spécial. sYN. } \\
\text { excentrique; original. } \\
\text { FORMES FaUTIVES } \\
\text { *en spécial. Anglicisme pour au rabais, soldé. } \\
\text { *livraison spéciale. Calque de " special delivery » pour livrai- } \\
\text { son par exprès. } \\
\text { *prix spécial. Anglicisme au sens de prix réduit. } \\
\text { *spécial. Anglicisme au sens de extraordinaire. Une assem- } \\
\text { blée extraordinaire (et non "spéciale). } \\
\text { *spécial du jour. Anglicisme pour plat du jour, menu du jour. } \\
\text { *spéciaux. Anglicisme au sens de soldes. }\end{array}$ & 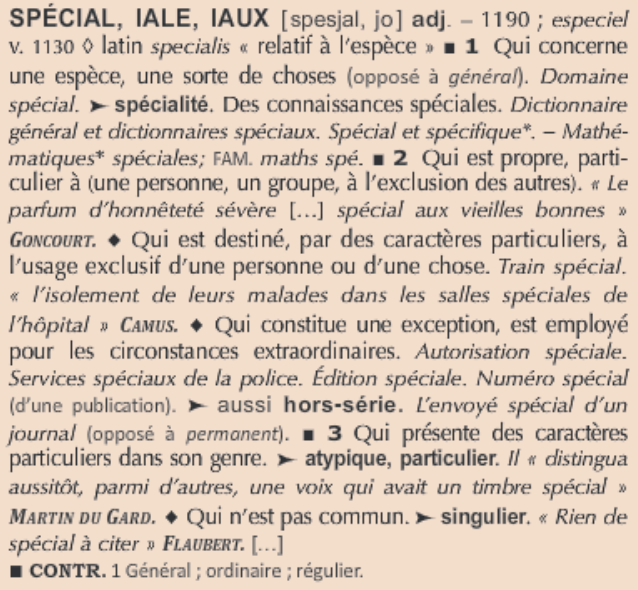 \\
\hline
\end{tabular}

Figure 1

Entrée du dictionnaire du mot SPÉCIAL ${ }^{1}$

Comme le laisse voir la richesse des informations lexicales contenues dans un article de dictionnaire, outre des activités de recherche usuelles en contextes de lecture ou d'écriture (orthographe d'un mot, recherche d'un sens, de synonymes, etc.), des usages que l'on qualifiera de plus « enrichis » peuvent être envisagés, telle la recherche de l'origine d'un mot, de ses antonymes, des cooccurrences dans lesquelles il apparait, etc.

Cet aperçu des connaissances et habiletés requises pour une consultation efficace des dictionnaires permet de comprendre que, sans une formation adéquate, les apprenants (et, parallèlement, les enseignants de langue), acquièrent difficilement ces connaissances et habiletés dictionnairiques (Bishop, 2000; Chi, 
2003; Fraser, 1999). Ainsi, on peut penser qu'un enseignant qui en sait davantage sur le contenu d'un article de dictionnaire et qui maitrise les abréviations qui y sont utilisées (par exemple, celles qui indiquent les registres de langue, incontournables dans l'apprentissage du français) sera mieux outillé pour transmettre ces connaissances à ses élèves, en conformité avec le contenu des programmes de formation, en particulier pour favoriser la consultation de l'outil en contexte de lecture et d'écriture.

\section{Utiliser le dictionnaire en contexte de lecture et d'écriture}

Les recherches qui se sont intéressées à l'impact du recours au dictionnaire lors de la lecture montrent que cet usage est susceptible de conduire à une plus grande augmentation du vocabulaire que lorsque le dictionnaire n'est pas mis à profit (Beech, 2004; Fraser, 1999; Laufer, 2000; Scott, Nagy et Flinspach, 2008). Ainsi, afin de rendre les élèves plus autonomes dans la compréhension du sens des mots nouveaux rencontrés au fil de leurs lectures, il importe de leur enseigner le plus tôt possible à recourir au dictionnaire (Biemiller, 2003; Giasson, 2011).

Parallèlement, en écriture, le dictionnaire peut soutenir l'apprenant durant tout le processus d'écriture : au moment de l'étape de planification, chez des élèves du primaire ou du secondaire, il pourra par exemple soutenir la création de banques de mots, ou l'exploration du vocabulaire relié à un thème (Tremblay, 2017). Durant l'étape de mise en texte ou de correction, il servira à vérifier l'orthographe d'un mot, à trouver des synonymes, à remplacer un anglicisme ou un mot de registre familier par le terme standard équivalent, etc. Enfin, lors de la phase de révision, qui consiste à améliorer le texte produit, il permettra de trouver un antonyme ou un mot dérivé pour remplacer un mot surutilisé, ou encore d'identifier une cooccurrence moins fréquente pour enrichir le vocabulaire du texte, telle l'utilisation des termes mener, conduire ou réaliser une recherche plutôt que de faire une recherche. Nous faisons l'hypothèse que si les enseignants font eux-mêmes usage du dictionnaire en contexte de lecture et d'écriture, ils seront plus susceptibles d'encourager cette pratique chez leurs élèves.

En somme, les connaissances des enseignants quant au dictionnaire, leurs attitudes motivationnelles, leurs sentiments envers cet outil et les contextes d'utilisation dans lesquels ils recourent eux-mêmes au dictionnaire représentent des informations à ce jour encore peu documentées. Pourtant, ces données constituent des variables importantes, susceptibles d'expliquer les pratiques d'enseignement et d'identifier les éléments de formation à intégrer aux cours de didactique du français dispensés dans les programmes de formation en enseignement, tant au primaire qu'au secondaire.

\section{Objectifs}

La présente étude entendait répondre à deux objectifs. Le premier consistait à caractériser la nature des sentiments (positifs et négatifs) des enseignants à l'égard du dictionnaire ainsi que leurs attitudes motivationnelles (sentiment de compétence et valeur attribuée à cet outil), selon le cycle d'enseignement et le type de dictionnaire (électronique ou papier). Le deuxième objectif était de décrire les dictionnaires détenus par les enseignants ainsi que les connaissances dictionnairiques et pratiques personnelles d'utilisation déclarées par ces derniers, en fonction du cycle d'enseignement et du type de dictionnaire. 


\section{Méthode}

Dans cette section, les différents aspects méthodologiques relatifs à l'étude seront rapportés : les participants et leur recrutement, la procédure et les mesures retenues.

\section{Participants et recrutement}

Pour répondre aux objectifs fixés, 300 enseignants (263 femmes et 37 hommes) du troisième cycle du primaire $(n=141)$, du premier cycle du secondaire $(n=73)$ et du deuxième cycle du secondaire $(n=86)$ ont été recrutés dans les 17 régions administratives du Québec. Le recrutement des participants a été effectué au moyen des réseaux sociaux et de contacts par courriel dans les commissions scolaires. Aucune compensation financière pour la participation à l'étude n'a été offerte de façon individuelle aux participants de l'étude, mais ceux-ci étaient admissibles à un tirage au sort (iPad, dictionnaires papier ou électronique). Au moment de leur participation à l'étude, les enseignants étaient âgés de 23 à 63 ans (moyenne $=39,15$; écart type $=8,05)$ et comptaient de 1 à 37 années d'expérience d'enseignement (moyenne $=13,86$; écart type $=6,79)$.

\section{Procédure et mesures}

Aux fins de l'étude, un questionnaire d'enquête en ligne a été élaboré à partir d'une adaptation des questionnaires de Geay (1995) et Gavriilidou (2013). Les mesures retenues pour notre étude sont consignées dans le tableau 1, dans lequel sont également rapportés la consistance interne des échelles de mesures utilisées et un exemple d'item.

\section{Tableau 1}

Sentiments à l'égard du dictionnaire et attitudes motivationnelles face au dictionnaire et à l'enseignement de son utilisation

\section{Mesures (nombre d'items) Alpha Exemple d'item}

\section{Sentiments à l'égard du dictionnaire papier}

Positifs (4) $\quad 0,88$ En général, pour vous, en termes de sentiments, utiliser le dictionnaire papier, c'est... amusant.

Négatifs (4) $\quad 0,88 \quad$ En général, pour vous, en termes de sentiments, utiliser le dictionnaire papier, c'est... ennuyant. 


\author{
Sentiments à légard du dictionnaire électronique \\ Positifs (4) $\quad 0,85$ En général, pour vous, en termes de sentiments, utiliser le dictionnaire électronique, c'est... \\ agréable. \\ Négatifs (4) $\quad 0,90 \quad$ En général, pour vous, en termes de sentiments, utiliser le dictionnaire électronique, c'est... \\ désagréable. \\ Attitudes motivationnelles face au dictionnaire et à l'enseignement de son utilisation \\ Sentiment de $\quad 0,89$ Je me sens compétent dans l'enseignement de l'utilisation du dictionnaire à mes élèves. \\ compétence (4) \\ Valeur (5) $\quad 0,82 \quad$ L'utilisation adéquate du dictionnaire permet une compréhension claire du fonctionnement de \\ la langue française.
}

Comme il est rapporté dans le tableau 1, les sentiments à l'égard du dictionnaire papier et électronique ont été mesurés à l'aide de quatre sous-échelles pour capter les sentiments positifs et négatifs à l'égard du dictionnaire papier, d'une part, et électronique, d'autre part. Les attitudes motivationnelles des enseignants ont quant à elles été évaluées par deux sous-échelles mesurant respectivement le sentiment de compétence et la valeur accordée au dictionnaire et à l'enseignement de son utilisation.

Le tableau 2 présente les mesures relatives aux connaissances déclarées par les enseignants, ainsi que leurs pratiques personnelles d'utilisation du dictionnaire (dictionnaires détenus, circonstances et buts d'utilisation).

\title{
Tableau 2
}

Dictionnaires détenus, connaissances déclarées, circonstances et buts d’utilisation

\begin{tabular}{lll}
\hline $\begin{array}{l}\text { Mesures } \\
\text { (nombre d'items) }\end{array}$ & Alpha & Exemple d'item \\
$\begin{array}{l}\text { Connaissances des dictionnaires } \\
\text { Types de dictionnaire (4) }\end{array}$ & s. $0 . \quad$ & $\begin{array}{l}\text { Je sais... Ce qu'est un dictionnaire étymologique et dans quel contexte } \\
\text { I'utiliser. }\end{array}$ \\
Abréviations (1) & s. 0. & $\begin{array}{l}\text { Je connais ou je comprends toutes les abréviations utilisées dans mon } \\
\text { dictionnaire. }\end{array}$ \\
Indications du registre (1) & s. 0. & $\begin{array}{l}\text { Pour voir comment un mot est utilisé dans le langage parlé, j'utilise les } \\
\text { indications de registre fournies dans l'entrée. }\end{array}$
\end{tabular}


Dictionnaires détenus par les enseignants

$\begin{array}{lll}\text { Dictionnaires papier (1) } & \text { S. o. } & \begin{array}{l}\text { Possédez-vous un ou plusieurs dictionnaires parmi les suivants: } \\ \text { Si oui, lesquels? }\end{array} \\ \text { Dictionnaires électroniques (1) } & \text { S. o. } & \begin{array}{l}\text { Possédez-vous un ou plusieurs dictionnaires parmi les suivants : } \\ \text { Si oui, lesquels? }\end{array}\end{array}$

Circonstances d'utilisation du dictionnaire papier

Lecture (1) S. o. En général, pour vous, en termes de circonstances, vous utilisez le

Écriture (1) S. o. En général, pour vous, en termes de circonstances, vous utilisez le dictionnaire papier pour... écrire un texte.

\section{Circonstances d'utilisation du dictionnaire électronique}
Lecture (1)
S. 0. En général, pour vous, en termes de circonstances, vous utilisez le dictionnaire électronique pour... lire un texte.
Écriture (1)
5. 0. En général, pour vous, en termes de circonstances, vous utilisez le dictionnaire électronique pour... écrire un texte.

\section{Buts d'utilisation personnelle du dictionnaire papier}

Buts enrichis (5) $\quad 0,85$ En général, pour vous, en termes de but, vous utilisez le dictionnaire papier pour... trouver des antonymes.

Buts usuels (4) 0,84 Eénéral, pour vous, en termes de but, vous utilisez le dictionnaire papier pour... trouver le sens d'un mot.

\section{Buts d'utilisation personnelle du dictionnaire électronique}

Buts enrichis (5) $\quad 0,90 \quad$ En général, pour vous, en termes de but, vous utilisez le dictionnaire électronique pour. . . trouver les dérivés d'un mot.

Buts usuels (4) $\quad 0,88$ En général, pour vous, en termes de but, vous utilisez le dictionnaire électronique pour... trouver des synonymes.

Comme il est rapporté dans le tableau 2, trois types de connaissances relatives au dictionnaire ont été mesurés : les différents types de dictionnaires, les abréviations qu'on y retrouve et les indications relatives au registre de langue. Pour l'ensemble des énoncés, les participants devaient indiquer leur degré d'accord à l'aide d'une échelle de Likert à six points allant de «fortement en désaccord » (1) à « fortement en accord» (6). Il est à noter que pour les connaissances des enseignants, aucun indice de consistance interne n'a été calculé puisqu'un seul item évaluait chacune de ces connaissances. En ce qui a trait aux pratiques personnelles d'utilisation du dictionnaire, elles ont été mesurées au moyen de 
trois indicateurs, soit les dictionnaires détenus à titre personnel, les circonstances d'utilisation et le type de buts d'utilisation du dictionnaire papier et électronique. Afin de connaitre les dictionnaires que les enseignants possèdent, les répondants devaient indiquer s'ils détenaient un ou plusieurs dictionnaires papier parmi les suivants: Petit Robert, Petit Larousse illustré, Multidictionnaire de la langue française, Hachette. Une question similaire a permis de connaitre les dictionnaires électroniques détenus ou consultés par les enseignants parmi les suivants: Trésor de la langue française informatisé, Petit Robert électronique, Antidote et Usito.

Deux circonstances d'utilisation des dictionnaires papier, d'une part, et électronique, d'autre part, ont été examinées : la lecture et l'écriture. Ainsi, pour chacun des énoncés proposés, les enseignants devaient indiquer à quelle fréquence ils recouraient au dictionnaire pour ces deux circonstances, en utilisant une échelle de Likert en 6 points, allant de « jamais » (1) à « chaque jour» (6).

Enfin, les buts d'utilisation du dictionnaire papier, d'une part, et électronique, d'autre part, ont chacun été mesurés à l'aide de neuf items que les enseignants devaient évaluer à l'aide d'une échelle de fréquence similaire à celle présentée précédemment. De manière exploratoire, des analyses factorielles ont été menées séparément pour les items relatifs à l'usage du dictionnaire papier et électronique, pour vérifier si des catégories d'items reflétaient différents types d'objectifs de consultation. Ce type d'analyse permet notamment de vérifier si les réponses aux différents items d'un questionnaire reflètent des patrons similaires, suggérant qu'ils mesurent des concepts communs plutôt que distincts, et qu'ils peuvent donc être regroupés plutôt que traités séparément les uns des autres (Field, 2013). Bien que les écrits théoriques ou les études empiriques ne distinguent pas formellement de types de pratiques d'utilisation du dictionnaire, notre analyse des réponses au questionnaire a permis de dégager deux catégories de pratiques. En effet, les résultats de ces analyses ont montré que pour les buts d'utilisation du dictionnaire papier, deux composantes ressortaient, avec des valeurs propres de 4,97 et 1,05 et expliquant 55,23\% et $11,67 \%$ de la variance. Pour les buts d'utilisation du dictionnaire papier, les résultats ont également généré deux composantes, avec des valeurs propres de 6,09 et 1,07 et expliquant 60,94\% et 10,72\% de la variance. Ces résultats montrent que, dans les deux cas, deux types de buts distincts peuvent être dégagés. À la lumière des items constitutifs de chacune des catégories, les catégories ont été libellées de la manière suivante : « buts d'utilisation usuels » et « buts enrichis ». En effet, utiliser le dictionnaire pour trouver l'orthographe d'un mot, trouver des synonymes, vérifier comment un mot est utilisé et trouver le sens d'un mot traduit des buts d'utilisation usuels. En contrepartie, le recours au dictionnaire pour trouver des antonymes, les origines d'un mot, les dérivés d'un mot, les familles de mots ou le sens d'une expression correspond plutôt à des buts d'utilisation enrichis.

\section{Résultats}

Afin de répondre aux objectifs de la recherche, les résultats sont divisés en deux parties. La première partie expose les résultats qui ont trait aux sentiments et attitudes motivationnelles des enseignants. La deuxième partie regroupe les résultats relatifs aux connaissances dictionnairiques déclarées par les enseignants, au nombre et au type de dictionnaires possédés à titre personnel ainsi qu'aux pratiques d'utilisation personnelle déclarées par les enseignants. En conformité avec les objectifs de l'étude, les résultats distingueront à la fois le cycle d'enseignement et le type de dictionnaire. 


\section{Les sentiments et les attitudes motivationnelles des enseignants}

Cette section présente les résultats relatifs aux sentiments à l'égard du dictionnaire et à leurs attitudes motivationnelles à l'égard de cet outil.

Les sentiments des enseignants envers les dictionnaires papier et électroniques

Les données relatives aux sentiments des enseignants à l'égard du dictionnaire ont été analysées à l'aide d'ANOVA menées séparément pour vérifier leur sentiment à l'égard du dictionnaire papier, d'une part, et électronique, d'autre part, incluant le cycle comme variable inter-sujets et le type de sentiment (positif ou négatif) comme variable intra-sujet. Ces résultats sont exposés dans les figures 2 et 3 cidessous.



Figure 2

Sentiments à l'égard du dictionnaire papier selon le niveau d'enseignement

Comme on peut le voir dans la figure 2, les enseignants manifestent significativement plus de sentiments positifs que négatifs à l'égard du dictionnaire papier $\left(F(1,289)=359,04, p<0,001, \mathrm{~h}^{2}=0,55\right)$, bien que cette différence soit moins marquée pour les enseignants du troisième cycle du primaire que pour les autres niveaux scolaires, comme l'indique l'interaction significative entre les sentiments et le niveau scolaire $\left(F(2,289)=6,10, p<0,003, \mathrm{~h}^{2}=0,04\right)$. 


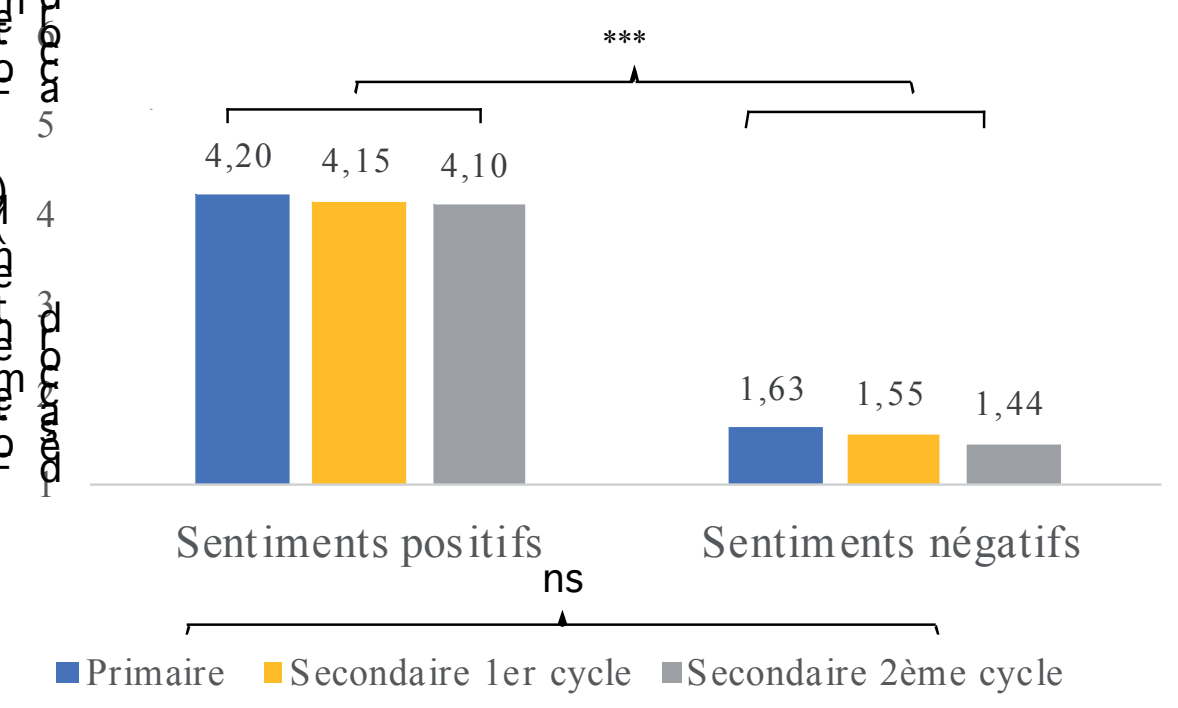

Figure 3

Sentiments à l'égard du dictionnaire électronique selon le niveau d'enseignement

Comme exposé dans la figure 3, le portrait est relativement similaire pour les sentiments à l'égard du dictionnaire électronique. En effet, les enseignants rapportent davantage de sentiments positifs que négatifs à l'égard du dictionnaire $\left(F(1,196)=593,21, p<0,001, \mathrm{~h}^{2}=0,75\right)$. Par contre, contrairement au sentiment à l'égard du dictionnaire papier, l'interaction entre le type de sentiment et le niveau scolaire n'est pas significative $\left(F(2,196)=0,06, p=0,941, \mathrm{~h}^{2}<0,01\right)$, révélant que la tendance des enseignants à éprouver davantage de sentiments positifs que négatifs ne varie pas selon le cycle d'enseignement.

\section{Attitudes motivationnelles des enseignants face au dictionnaire}

La figure 4 présente à la fois le sentiment de compétence que les enseignants déclarent relativement à leur connaissance et à leur usage de l'outil, ainsi que la valeur qu'ils attribuent à l'ouvrage, selon le cycle d'enseignement. Cependant, contrairement aux autres mesures, ces variables concernent le dictionnaire en général, sans égard à sa forme papier ou électronique. Une analyse de variance multivariée (MANOVA) prédisant le sentiment de compétence et la valeur et incluant le niveau scolaire comme variable inter-sujets a été réalisée afin d'examiner les différences dans ces indicateurs motivationnels selon le cycle d'enseignement. 
6

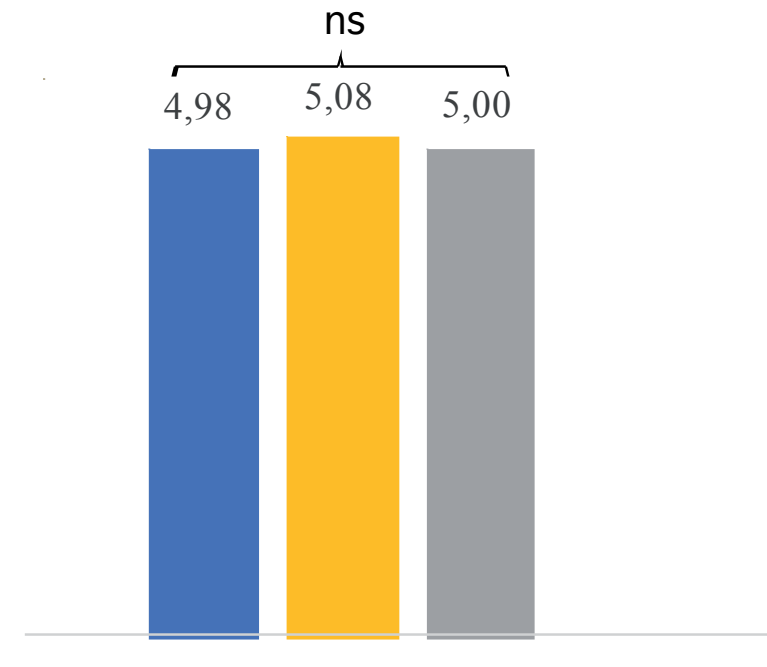

\section{Sentiment de compétence \\ - Primaire $\square$ Secondaire 1er cycle $\square$ Secondaire 2ème cycle}

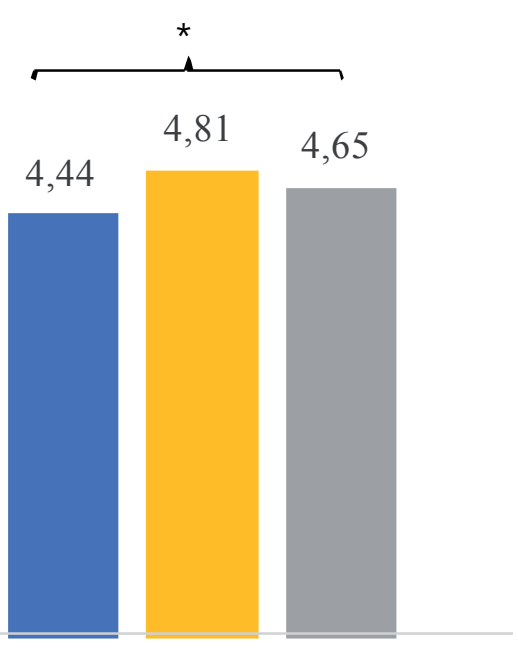

\section{Figure 4}

Attitudes motivationnelles des enseignants face au dictionnaire selon le niveau d'enseignement

Les résultats ont révélé un effet significatif du niveau scolaire $\left(F(4,594)=2,69, p=0,031, \eta^{2}=0,02\right)$. L'examen subséquent des tests univariés indique que le sentiment de compétence des enseignants ne varie pas significativement selon le cycle d'enseignement $\left(F(2,297)=0,33, p=0,716, \eta^{2}<0,01\right)$. Par contre, une différence significative selon le niveau scolaire a été obtenue pour la valeur attribuée au dictionnaire $\left(F(2,297)=5,35, p=0,005, \eta^{2}=0,04\right)$. Des tests de contrastes ont indiqué que les enseignants du primaire accordent significativement moins de valeur au dictionnaire que ceux du premier cycle du secondaire $(p=0,002)$ et marginalement moins que ceux du deuxième cycle du secondaire $(p=0,058)$.

\section{Connaissances, dictionnaires détenus et pratiques personnelles d'utilisation du dictionnaire}

Cette section présente les résultats concernant les connaissances relatives au dictionnaire, les différents types de dictionnaires détenus par les enseignants à titre personnel et l'utilisation personnelle que les enseignants déclarent faire du dictionnaire en contexte de lecture et d'écriture.

\section{Connaissances déclarées des différents types de dictionnaires}

Deux types de connaissances sur le dictionnaire ont été mesurés : les connaissances des différents types de dictionnaires, d'une part, et la connaissance des abréviations et de l'indication du registre dans un article de dictionnaire, d'autre part. 
Les connaissances déclarées par les enseignants quant aux différents types de dictionnaires (étymologique, général, bilingue, spécialisé) ont été analysées à l'aide d'une ANOVA à mesures répétées, incluant le type de dictionnaires comme variable intra-sujet et le niveau scolaire comme variable inter-sujets. Ces résultats sont présentés dans la figure 5 .



- Primaire $\quad$ Secondaire 1er cycle $\quad$ Secondaire 2ème cycle

Figure 5

Connaissances déclarées des différents types de dictionnaires selon le niveau

Les données ont révélé que l'ampleur des connaissances différait selon le type de dictionnaires et le niveau scolaire, comme en témoigne l'interaction significative entre ces deux variables $(F(6,592)=9,26$, $\left.p<0,001, \eta^{2}=0,09\right)$. Pour décomposer cette interaction, une MANOVA a été effectuée afin de prédire la connaissance relative à chacun des quatre types de dictionnaires, selon le niveau scolaire. Les résultats montrent que la connaissance des enseignants diffère selon le cycle d'enseignement pour les dictionnaires étymologiques $\left(F(2,297)=26,94, p<0,001, \eta^{2}=0,15\right)$, technique $\left(F(2,297)=15,77, p<0,001, \eta^{2}=\right.$ $0,10)$, et marginalement pour le dictionnaire général $\left(F(2,297)=2,95, p=0,054, \eta^{2}=0,02\right)$. Par contre, la connaissance du dictionnaire bilingue ne diffère pas selon le cycle d'enseignement $(F(2,297)=1,16$, $\left.p=0,314, \eta^{2}<0,01\right)$. Des tests de contrastes subséquents ont montré que les enseignants du troisième cycle du primaire ont une connaissance moindre des dictionnaires étymologique et technique que les enseignants des premier et deuxième cycles du secondaire. Enfin, leur connaissance du dictionnaire général est moins étendue que celle des enseignants du deuxième cycle du secondaire, mais pas du premier cycle du secondaire. 
Deux types de connaissances relatives à l'utilisation du dictionnaire ont été examinés : la connaissance des abréviations utilisées dans les articles de dictionnaire et les indications relatives au registre de langue fournies dans une entrée de dictionnaire. Les résultats sont exposés dans la figure 6.

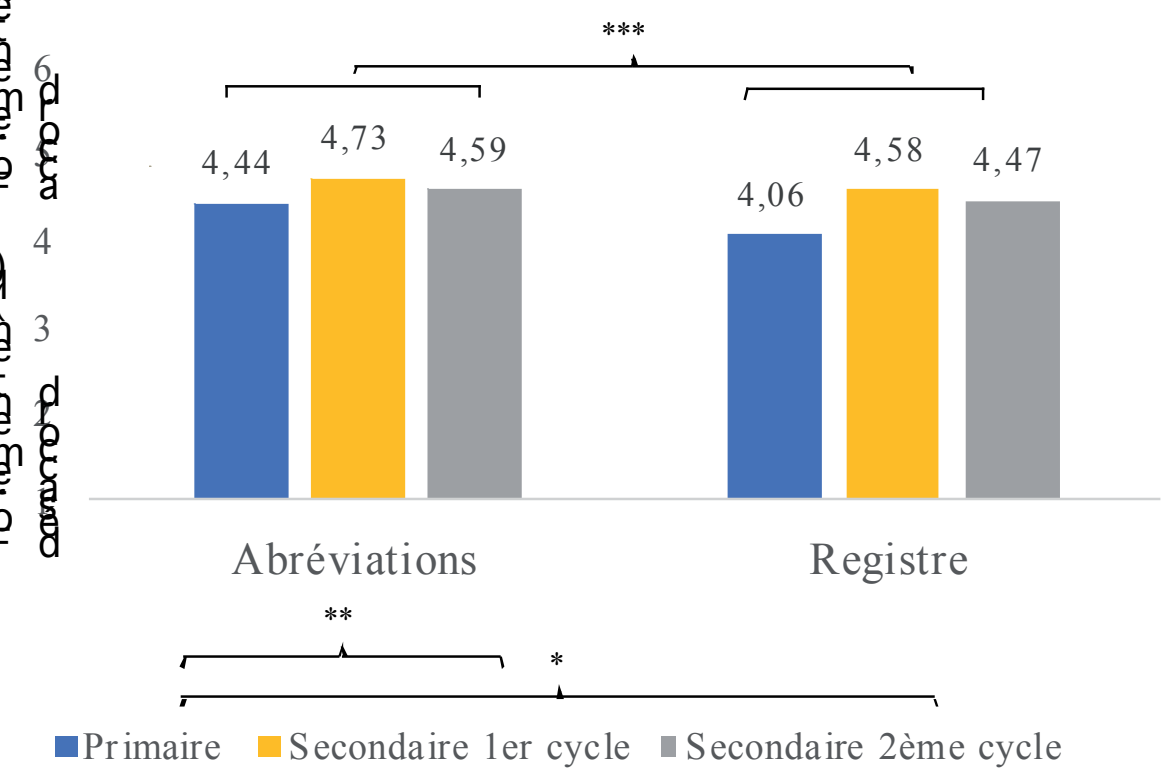

Figure 6

Connaissances sur le dictionnaire selon le niveau

Afin de déterminer si le degré de maitrise de ces deux connaissances variait significativement selon le cycle d'enseignement, une ANOVA incluant le type de connaissances comme variable intra-sujet et le niveau d'enseignement comme variable inter-sujets a été menée. Les résultats de l'analyse révèlent que la connaissance des abréviations est significativement mieux maitrisée que celle ayant trait au registre $\left(F(1,297)=5,27, p=0,002, \eta^{2}=0,02\right)$. De plus, l'effet significatif du niveau scolaire $(F(2,297)=4,46$, $\left.p=0,012, \eta^{2}=0,03\right)$ indique des différences significatives dans le degré de maitrise selon le niveau scolaire. Des tests de contrastes précisent que les enseignants du troisième cycle du primaire maitrisent significativement moins ces connaissances que ceux du premier cycle du secondaire $(p=0,006)$ et du deuxième cycle du secondaire $(p=0,043)$.

\section{Quels dictionnaires les enseignants possèdent-ils?}

Le tableau 4 présente le nombre et le type de dictionnaires détenus (ou consultés, dans le cas de certains dictionnaires électroniques en ligne) par les enseignants, qu'il s'agisse de dictionnaires papier ou électroniques. Des ANOVA ont ensuite été menées pour vérifier si la proportion de chacun des titres variait selon le cycle d'enseignement. Les résultats de ces ANOVA sont inclus dans le tableau 4. Enfin, des tests- $t$ appariés, dont le seuil de signification a été ajusté pour la répétition des tests, ont été menés pour vérifier si, pour chacun des cycles, la proportion de dictionnaires détenus par les enseignants 
variait significativement. Les moyennes qui diffèrent significativement sont accompagnées d'un indice dans le tableau 4.

Pour faciliter la compréhension des résultats, les résultats concernant les dictionnaires papier et électroniques sont détaillés séparément dans les prochaines sections.

\section{Tableau 4}

Pourcentages de dictionnaires détenus ou consultés par les enseignants selon le type

\begin{tabular}{|c|c|c|c|c|}
\hline & $\begin{array}{l}3 \text { cycle du } \\
\text { primaire }\end{array}$ & $\begin{array}{l}1 \text { cycle du } \\
\text { secondaire }\end{array}$ & $\begin{array}{l}2 \text { cycle du } \\
\text { seconda ire }\end{array}$ & Résultat de l'ANOVA selon le cycle \\
\hline \multicolumn{5}{|l|}{ Dictionnaire papier } \\
\hline $\begin{array}{l}\text { Le petit Robe it } \\
\text { Le Larousse illus tré }\end{array}$ & $\begin{array}{l}65,20 \% \%_{a} \\
82,30 \%\end{array}$ & $\begin{array}{l}79,50 \% \\
84,90 \%\end{array}$ & $\begin{array}{l}90,70 \% \mathrm{a} \\
82,60 \%\end{array}$ & $\begin{array}{l}F(2,297)=10,38, p<0,001, \eta^{2}=0,07 \\
F(2,297)=0,128, p=0,880, \eta^{2}<0,01\end{array}$ \\
\hline $\begin{array}{l}\text { Le Multidictionnaire } \\
\text { Le Hachette } \\
\text { Aucun }\end{array}$ & $\begin{array}{c}68,10 \% \mathrm{~b}, \mathrm{c} \\
9,20 \% \\
0 \%\end{array}$ & $\begin{array}{c}90,40 \% \text { b } \\
15,10 \% \\
0 \%\end{array}$ & $\begin{array}{c}83,70 \% \%_{\mathrm{c}} \\
8,10 \% \\
0 \%\end{array}$ & $\begin{array}{c}\mathrm{F}(2,297)=8,516, \mathrm{p}<0,001, \eta^{2}=0,05 \\
\mathrm{~F}(2,297)=1,199, \mathrm{p}=0,303, \eta^{2}<0,01 \\
\mathrm{~N} / \mathrm{A}\end{array}$ \\
\hline \multicolumn{5}{|l|}{ Dictionnaire électronique } \\
\hline Tré sor de la langue fra nçaise & $1,40 \%$ & $0 \%$ & $2,30 \%$ & N/A \\
\hline Petit Robert électroni que & $\begin{array}{c}5,70 \% \\
46,10 \%\end{array}$ & $\begin{array}{l}12,30 \% \\
47,90 \%\end{array}$ & $\begin{array}{c}8,10 \% \\
42,30 \%\end{array}$ & $\begin{array}{l}\mathrm{F}(2,297)=0,815, \mathrm{p}=0,444, \eta^{2}<0,01 \\
\mathrm{~F}(2,297)=1,448, \mathrm{p}=0,237, \eta^{2}=0,01\end{array}$ \\
\hline Usito & $5,70 \%$ & $6,80 \%$ & $8,10 \%$ & $\mathrm{~F}(2,297)=0,415, \mathrm{p}=0,661, \eta^{2}<0,01$ \\
\hline Aucun & $36,90 \%$ & $35,60 \%$ & $43 \%$ & $F(2,297)=0,261, p=0,770, \eta^{2}<0,01$ \\
\hline
\end{tabular}

Comme il est exposé dans le tableau 4, la proportion de dictionnaires papier détenus par les enseignants selon les cycles varie. Spécifiquement, en comparaison avec les enseignants du troisième cycle du primaire, ceux du deuxième cycle du secondaire sont plus nombreux à détenir un Petit Robert. En contrepartie, le Multidictionnaire est plus courant chez les enseignants du premier et du deuxième cycle du secondaire que chez ceux du troisième cycle du primaire. Enfin, la popularité du Larousse et du Hachette ne varie pas significativement selon le cycle scolaire.

Par ailleurs, pour vérifier si, pour chacun des trois cycles d'enseignement, la proportion de chacun des dictionnaires détenus différait significativement, des ANOVA subséquentes ont été menées séparément pour chacun des cycles d'enseignement. Pour le troisième cycle du primaire, le résultat de l'ANOVA s'est révélé significatif $\left(F(3,138)=148,78, p<0,001, \eta^{2}=0,76\right)$. Des tests- $t$ appariés, dont le seuil de signification a été corrigé pour la répétition des tests, ont révélé que le Larousse illustré est significativement plus fréquent que le Petit Robert et le Hachette, mais pas que le Multidictionnaire.

Pour le premier cycle du secondaire, le résultat de l'ANOVA s'est révélé significatif $(F(3,70)=85,35$, $\left.p<0,001, \eta^{2}=0,79\right)$. Des tests- $t$ appariés, dont le seuil de signification a été corrigé pour la répétition des tests, ont révélé que le dictionnaire le plus fréquemment possédé par les enseignants est le Multidictionnaire. Par contre, les enseignants ne détiennent pas significativement plus de Multidictionnaire que de Petit Robert ou de Larousse illustré. Cela dit, le Hachette est significativement moins détenu que les trois autres ouvrages.

Pour le deuxième cycle du secondaire, le résultat d'une ANOVA s'est révélé significatif $(F(3,83)=168,11$, $p<0,001, \eta^{2}=0,86$. Des tests- $t$ appariés, dont le seuil de signification a été corrigé pour la répétition des tests, ont montré qu'au deuxième cycle du secondaire, le dictionnaire le plus populaire chez les 
enseignants est le Petit Robert. À nouveau, le pourcentage plus élevé de Petit Robert ne diffère pas significativement ni du Larousse illustré ni du Multidictionnaire. Enfin, le Hachette est significativement moins détenu que les trois autres ouvrages.

En ce qui concerne l'usage des dictionnaires électroniques, les données du tableau 4 révèlent d'abord que pour chacun des trois cycles, plus de $35 \%$ des enseignants ne possèdent pas ou ne consultent pas de dictionnaires électroniques, parmi ceux mentionnés dans le questionnaire. De plus, peu importe le cycle, le dictionnaire Antidote est le plus populaire.

À nouveau, des ANOVA supplémentaires ont été menées pour déterminer si la popularité des dictionnaires pour un même cycle variait significativement. Au troisième cycle du primaire, le résultat a montré une différence significative selon les dictionnaires électroniques détenus par les enseignants $\left(F(4,137)=118,80, p<0,001, \eta^{2}=0,78\right)$. Le résultat de tests- $t$ appariés dont le seuil de signification a été corrigé pour la répétition des tests a révélé que le dictionnaire Antidote est significativement plus répandu auprès des enseignants que les trois autres dictionnaires électroniques présentés.

Au premier cycle du secondaire,l'ANOVA a également révélé des différences selon le dictionnaire détenu $\left(F(4,69)=99,88, p<0,001, \eta^{2}=0,85\right)$. À nouveau, des tests- $t$ appariés dont le seuil de signification a été corrigé pour la répétition des tests ont révélé que le dictionnaire Antidote est significativement plus répandu auprès des enseignants du premier cycle du secondaire que les trois autres dictionnaires électroniques présentés.

Enfin, au deuxième cycle du secondaire, des différences significatives selon le dictionnaire détenu ont également été observées $\left(F(4,82)=120,90, p<0,001, \eta^{2}=0,86\right)$. Encore une fois, des tests- $t$ appariés, dont le seuil de signification a été corrigé pour la répétition des tests, ont révélé que le dictionnaire Antidote est significativement plus répandu auprès des enseignants du deuxième cycle du secondaire que les trois autres dictionnaires électroniques présentés.

\section{Circonstances d'utilisation du dictionnaire}

Une analyse ANOVA a été effectuée pour vérifier si le dictionnaire est davantage utilisé pour la lecture ou pour l'écriture, d'une part, et si ces circonstances varient selon le type de dictionnaire (papier vs électronique). Dans cette analyse, la circonstance d'utilisation (lire vs écrire) et le type de dictionnaires (papier vs électronique) ont été inclus comme variables intra-sujet, alors que le cycle d'enseignement a été inclus comme variable inter-sujets. Les résultats, présentés dans la figure 7, ont révélé une interaction significative entre la circonstance d'utilisation des dictionnaires (papier et électroniques confondus) et le niveau scolaire $\left(F(2,188)=8,04, p<0,001, \eta^{2}=0,08\right)$. La décomposition de cet effet a révélé un effet significatif du niveau scolaire uniquement pour la lecture, mais pas pour l'écriture, révélant que les enseignants du troisième cycle du primaire recourent moins au dictionnaire pour la lecture. Ainsi, bien que le dictionnaire soit plus utilisé pour l'écriture que pour la lecture pour les différents niveaux scolaires examinés, la différence entre la fréquence d'utilisation pour les deux circonstances est plus marquée au troisième cycle du primaire que pour les autres cycles d'enseignement. Tous les autres effets principaux ou d'interaction se sont avérés non significatifs (tous les $p>0,28)^{2}$. 


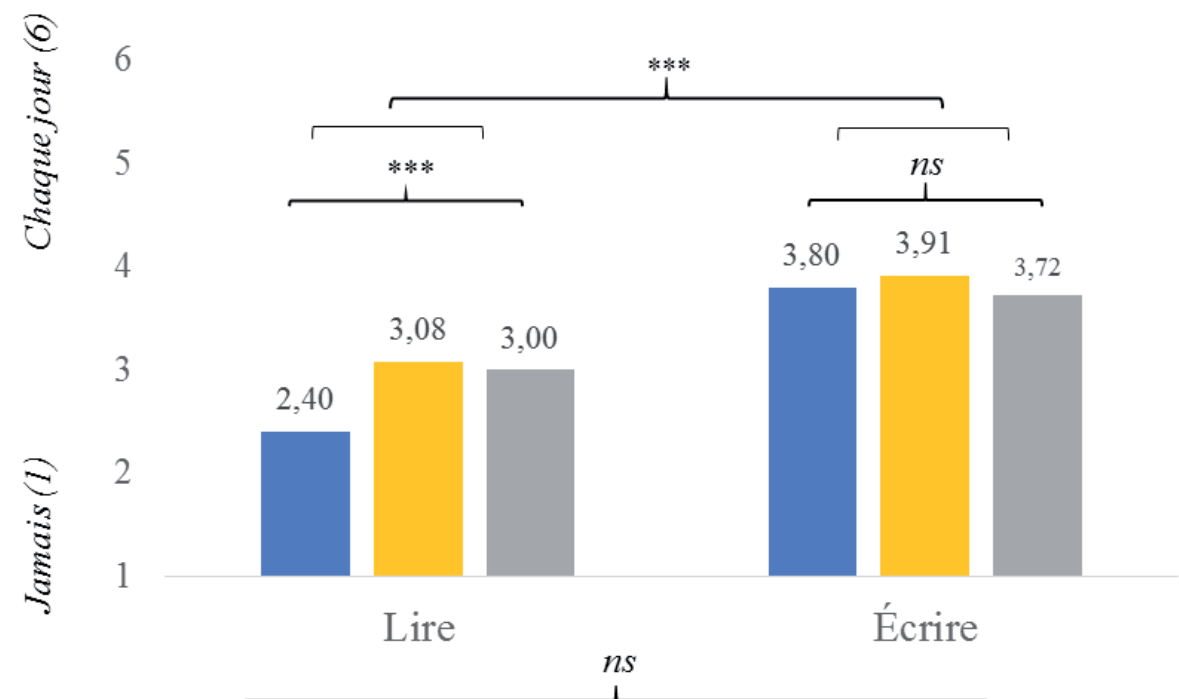

घrimaire $\square$ Secondaire 1er cycle $\square$ Secondaire 2ème cycle

Figure 7

Circonstances d'utilisation du dictionnaire selon le niveau

Afin de comparer les buts d'utilisation des dictionnaires papier et électronique des enseignants selon le niveau scolaire, une ANOVA à mesures répétées a été réalisée, incluant le type de dictionnaires (papier vs électroniques) et le type de buts déclarés (usuel vs enrichi) comme variable intra-sujet et le cycle d'enseignement comme variable inter-sujets. Les résultats ont révélé que, tous types de dictionnaires et niveaux confondus, les enseignants rapportent davantage de buts usuels qu'enrichis $(F(1,188)=469,84$, $\left.p<0,001, \eta^{2}=0,71\right)$. De plus, ces résultats ne varient pas selon le type de dictionnaires utilisé, ni selon le niveau, tel que l'indique l'interaction non significative entre le type de buts et le type de dictionnaires $\left(F(1,188)=1,13, p=0,288, \eta^{2}<0,01\right)$, ainsi qu'entre le type de buts et le niveau scolaire $\left(F(2,188)=0,91, p=0,406, \eta^{2}=0,01\right)^{3}$. Les données relatives aux buts d'utilisation du dictionnaire papier et électronique sont présentées dans la figure 8 ci-dessous. 


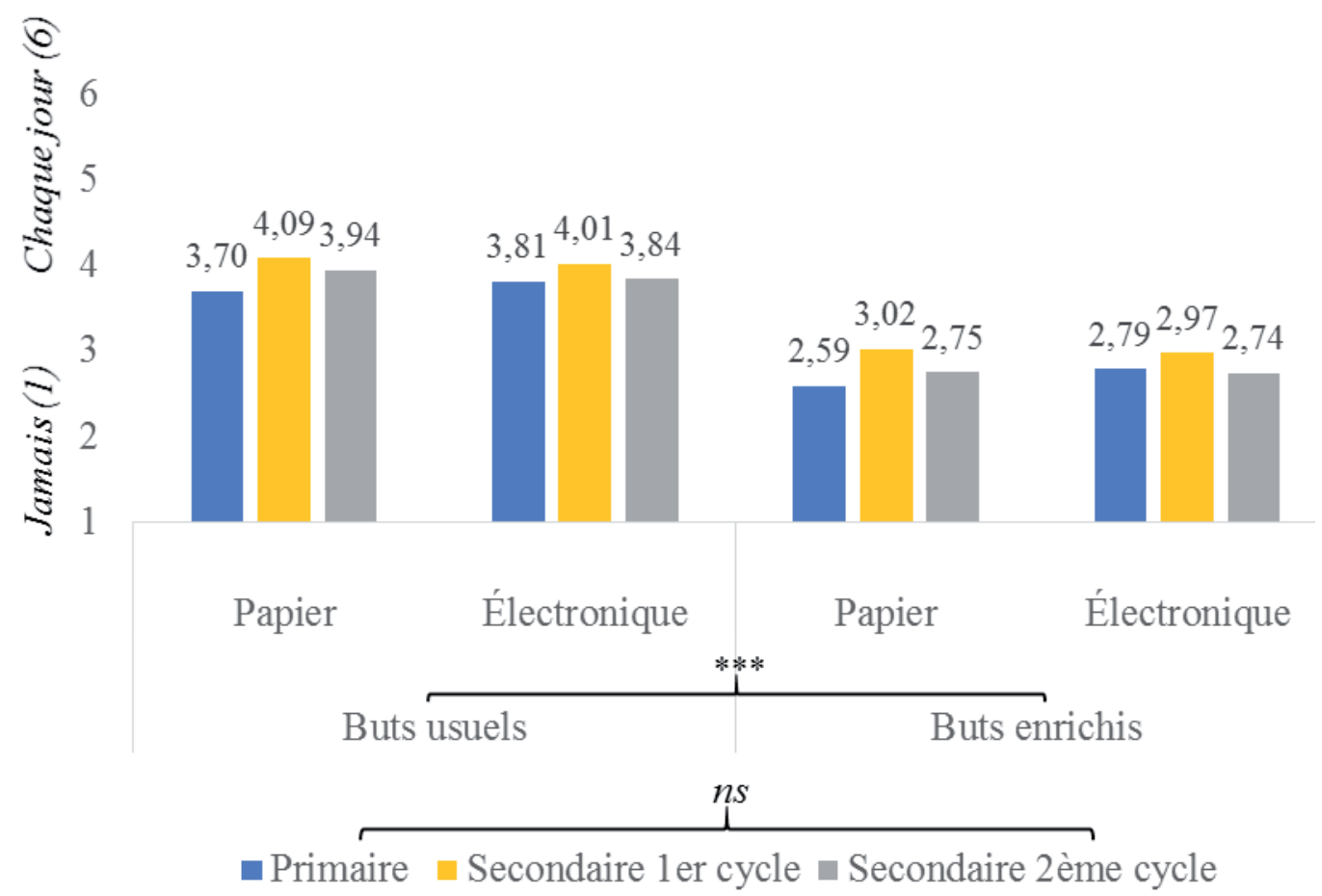

Figure 8

Buts personnels lors de l'utilisation du dictionnaire papier ou électronique

\section{Discussion}

La présente étude entendait évaluer la nature des sentiments (positifs et négatifs) et les attitudes motivationnelles (sentiment de compétence et valeur attribuée) envers le dictionnaire, d'une part, et décrire les connaissances déclarées de cet outil, les dictionnaires détenus et les pratiques personnelles d'utilisation des enseignants, d'autre part, selon leur cycle d'enseignement et le type de dictionnaires. Dans les prochaines sections, nous commenterons les résultats les plus saillants relativement aux différentes variables examinées.

\section{Des attitudes motivationnelles et sentiments positifs à l'égard du dictionnaire}

Les données consignées dans la présente étude ont révélé que, dans l'ensemble, les enseignants ont des attitudes motivationnelles et des sentiments relativement positifs envers le dictionnaire, peu importe leur cycle d'enseignement et le type de dictionnaires pris en compte. Ce résultat est de bon augure puisque, comme l'ont montré certaines études (par exemple, Holzberger et al., 2013; Moè et al., 2010), des attitudes positives jouent un rôle dans l'adoption de pratiques pédagogiques plus efficaces. On peut donc penser que les enseignants accueilleront positivement d'éventuelles mesures de perfectionnement portant sur le dictionnaire et ses usages, en vue d'améliorer leurs pratiques d'enseignement de la langue. 
Nos résultats montrent également que les enseignants du secondaire rapportent généralement des sentiments plus positifs envers le dictionnaire que ceux du primaire, ce qui peut s'expliquer par le fait que ces enseignants sont spécialistes du français et de son enseignement alors que ceux du primaire sont généralistes. De plus, si l'ensemble des enseignants de l'échantillon possède un sentiment de compétence relativement élevé quant à l'utilisation du dictionnaire et qu'ils y accordent une valeur également assez élevée, cette dernière est accrue chez les enseignants du secondaire.

\section{Des connaissances dictionnairiques relativement limitées}

Par ailleurs, les enseignants connaissent assez bien les dictionnaires de type général et bilingue, les dictionnaires les plus courants, alors que leur connaissance des dictionnaires de type étymologique et technique est beaucoup moins étendue, surtout pour les enseignants qui œuvrent au troisième cycle du primaire. De plus, la connaissance des dictionnaires est généralement meilleure chez les enseignants qui interviennent auprès des élèves du deuxième cycle du secondaire, ce qui n'est pas étonnant, au vu de l'exigence d'un enseignement approfondi de la langue à ce niveau scolaire et les prescriptions ministérielles du programme du secondaire (MELS, 2011b). En ce qui a trait aux abréviations et à l'indication du registre, il est d'abord surprenant de constater que ces connaissances ne soient pas mieux maitrisées, considérant qu'il s'agit de connaissances de base nécessaires à la compréhension du contenu d'un article de dictionnaire (Fuertes-Olivera et Tarp, 2011; Lew, 2013; Scholfield, 1999), et ce, aussi bien au primaire (MELS, 2011a) qu'au secondaire (MELS, 2011b). De plus, les résultats ont montré que les enseignants du secondaire maitrisaient davantage ces connaissances que ceux du primaire, ce qui peut s'expliquer encore une fois par le fait qu'ils sont davantage spécialistes de la langue que leurs collègues du primaire.

Somme toute, ces résultats sont plutôt encourageants et soulignent la valeur attribuée au dictionnaire par les enseignants du primaire comme du secondaire. Cependant, les connaissances des enseignants concernant cet outil apparaissent relativement limitées, ce qui confirme les besoins de formation continue dans ce domaine, non seulement au primaire et au secondaire, mais aussi au collégial (CaronBouchard, Pronovost, Quesnel, Perrault et Deslauriers, 2011) et à l'université (Bernier, 2010). Il est également intéressant de constater que le type de dictionnaires, soit papier ou électronique, ne constitue pas un enjeu dans les attitudes envers cet outil.

\section{Des pratiques d'utilisation personnelles du dictionnaire variées}

Les résultats concernant les pratiques personnelles d'utilisation du dictionnaire révèlent d'abord que, dans l'ensemble, les enseignants possèdent une variété d'ouvrages, laissant croire qu'ils sont au fait que chaque dictionnaire est susceptible de répondre à des besoins communicationnels et linguistiques distincts (Nesi, 1999). Plus spécifiquement, les spécialistes de français du deuxième cycle du secondaire sont plus nombreux à posséder un dictionnaire Petit Robert comparativement aux enseignants du primaire alors que le Multidictionnaire est plus répandu tout au long du secondaire comparativement au troisième cycle du primaire. Ces résultats sont intéressants puisqu'ils reflètent les usages enrichis que les enseignants de la fin du secondaire sont appelés à enseigner à leurs élèves et qui sont facilités par le Petit Robert en comparaison avec le Multidictionnaire, tel que le montre l'analyse comparative d'articles tirés de ces deux dictionnaires, dans la figure 1 plus haut. Le Larousse, un dictionnaire contenant beaucoup d'images, est quant à lui le plus populaire auprès des enseignants du troisième cycle. 
Par ailleurs, nos données indiquent qu'un peu moins de $65 \%$ de notre échantillon dispose d'un dictionnaire électronique, et il s'agit le plus souvent d'Antidote. Le grand nombre d'enseignants qui ne dispose pas de dictionnaire électronique pourrait s'expliquer par les coûts rébarbatifs de ce type de ressources (pour les versions numériques de dictionnaires, tels le Petit Robert électronique, Usito, Antidote) ou le manque d'intérêt envers celle-ci (pour les enseignants qui disent ne pas utiliser les ressources en ligne, comme le TLFi). Enfin, on peut faire l'hypothèse que certains enseignants sont réfractaires à l'usage des technologies numériques ou qu'ils ne disposent pas des compétences numériques nécessaires pour la consultation d'un dictionnaire électronique. À cet égard, alors que le nombre d'environnements éducatifs dans lesquels chaque élève est équipé d'un ordinateur portable ou d'une tablette électronique ne cesse de croitre (Karsenti et Collin, 2013), des recherches récentes indiquent que les enseignants ne maitrisent encore que faiblement la compétence à intégrer les TIC (Stockless et Beaupré, 2014; Villeneuve, Karsenti, Raby et Meunier, 2012). En ce sens, des efforts pour favoriser l'accessibilité aux dictionnaires électroniques, comme mesure d'accompagnement de la lecture et de l'écriture en contexte numérique, devraient être entrepris par le Ministère et les responsables de l'attribution de matériel pédagogique.

Les résultats concernant les circonstances d'utilisation du dictionnaire indiquent quant à eux que, peu importe le cycle d'enseignement, les enseignants utilisent plus fréquemment le dictionnaire pour l'écriture que pour la lecture. Ce résultat était prévisible puisque le dictionnaire permet de répondre à plusieurs besoins chez le scripteur (Tremblay, 2017), mais à un seul besoin chez le lecteur, soit celui de trouver le sens d'un mot (Giasson, 2011). Enfin, les enseignants du primaire utilisent significativement moins le dictionnaire en situation de lecture que leurs collègues du secondaire. Une hypothèse possible (bien que non documentée, à notre connaissance) pour expliquer ce constat serait que les enseignants du secondaire, spécialistes du français, sont susceptibles de lire davantage que les autres et donc de recourir plus fréquemment au dictionnaire.

Pour terminer, nos résultats ont montré que lorsqu'ils utilisent un dictionnaire, les enseignants rapportent généralement des buts d'utilisation qui reflètent des pratiques que nous avons qualifiées d'usuelles, en opposition à des pratiques qui exploitent le dictionnaire dans une perspective " enrichie ». Ces données soulignent non seulement le manque de connaissances des enseignants quant au potentiel du dictionnaire comme outil d'aide à l'écriture (Bishop, 2000; Carstens, 1995; Tremblay, 2017), mais corroborent également les lacunes des enseignants relatives à la connaissance des abréviations, des indications du registre et des types de dictionnaires. À nouveau, ces données mettent en lumière la nature des besoins de formation continue des enseignants, tant du primaire que du secondaire, à propos du potentiel des dictionnaires papier et électroniques comme outils de développement des connaissances langagières (Beech, 2004; Bernier, 2010).

\section{Limites et pistes de formation et de recherche futures}

Cette étude comporte certaines limites, qu'il convient ici de reconnaitre et qui pourront guider la recherche future sur le sujet. D'abord, les enseignants de l'échantillon ont été recrutés sur une base volontaire si bien que les données se généralisent exclusivement à des enseignants qui partagent les mêmes caractéristiques que ceux de l'échantillon. De plus, la nature autorapportée des données est susceptible de comporter certains biais, notamment de désirabilité sociale (Vallerand, 2006), qui 
pourraient avoir incité les enseignants à rapporter des pratiques plus positives que celles qui sont réellement mobilisées. Par exemple, il est possible que les enseignants aient surestimé, consciemment ou non, la qualité ou la fréquence de leurs pratiques d'utilisation des dictionnaires. Néanmoins, le fait que les buts d'utilisation rapportés reflètent davantage des pratiques usuelles qu'enrichies donne à penser qu'en dépit de ces biais potentiels, les pratiques rapportées traduisent adéquatement certaines lacunes. Pour contourner les limites rapportées, il serait souhaitable d’obtenir des données provenant d'observations directes, par exemple, en évaluantla qualité des stratégies de recherches dans le dictionnaire dans un contexte expérimental, afin de corroborer les résultats de la présente étude. Des observations directes lorsque les enseignants sont placés en contexte de lecture et d'écriture procureraient en ce sens une mesure plus objective de leur propension à recourir aux différents dictionnaires et à quelles fins. Enfin, une autre limite concerne le manque d'informations relatives à certaines caractéristiques des milieux d'enseignement dans lesquels œuvraient les enseignants de l'échantillon et qui pourraient avoir modulé les résultats, tels le statut public ou privé de l'institution scolaire ou encore son milieu socioéconomique.

\section{Conclusion}

La présente étude fournit des données empiriques novatrices qui documentent le type de sentiment et les attitudes motivationnelles des enseignants envers le dictionnaire, d'une part, et leurs connaissances et pratiques d'utilisation personnelles relatives à cet outil, d'autre part. En plus de souligner les perceptions positives associées à cette ressource, les résultats ont mis en lumière certaines lacunes, notamment en ce qui concerne l'accès aux dictionnaires électroniques ou encore aux buts d'utilisation des différents dictionnaires, qui demeurent plutôt traditionnels. Ces derniers résultats révèlent certains besoins de formation des enseignants du primaire et du secondaire afin qu'ils puissent tirer profit de cet outil incontournable dans l'apprentissage et l'enseignement de la langue française.

\section{Références}

Anctil, D. (2010). L'erreur lexicale au secondaire : analyse d'erreurs lexicales d'élèves de $3^{e}$ secondaire et description du rapport à l'erreur lexicale d'enseignants de français (Thèse de doctorat, Université de Montréal). Repéré à http://hdl.handle.net/1866/5077

Bandura, A. (1977). Self-efficacy: Toward a unifying theory of behavioral change. Psychological Review, 84(2), 191-215. http://dx.doi.org/10.1037/0033-295x.84.2.191

Baumeister, R. F. et Bushman, B. J. (2007). Angry emotions and aggressive behaviors. Dans G. Steffgen et M. Gollwitzer (dir.), Emotions and aggressive behavior (p. 61-75). Ashland, OH : Hogrefe \& Huber.

Beech, J. R. (2004). Using a dictionary: its influence on children's reading, spelling, and phonology. Reading Psychology, 25(1), 19-36. http://dx.doi.org/10.1080/02702710490271819

Bernier, M. (2010). Effet d'une formation à l'utilisation d'aides logicielles sur des scripteurs francophones de premier cycle universitaire. Revue internationale des technologies en pédagogie universitaire, 7(3), 60-72. http://dx.doi.org/10.7202/1003564ar

Biemiller, A. (2003). Vocabulary: Needed if more children are to read well. Reading Psychology, 24(3/4), 323-335. http:// dx.doi.org/10.1080/02702710390227297

Bishop, G. (2000). Developing learner strategies in the use of dictionaries as a productive language learning tool. The Language Learning Journal, 22(1), 58-62. http://dx.doi.org/10.1080/09571730085200261 
Caron-Bouchard, M., Pronovost, M., Quesnel, C., Perrault, C. et Deslauriers, K. (2011). Outils virtuels et qualité de la langue. Repéré à https://cdc.qc.ca/parea/787900-caron-bouchard-et-al-outils-virtuel-qualite-langue-brebeufPAREA-2011.pdf

Carstens, A. (1995). Language teaching and dictionary use: An overview. Lexikos, 5, 105-116. http://dx.doi.org/10.5788/5-1-1059

Chartrand, S.-G. et Émery-Bruneau, J. (2013). Caractéristiques de 50 genres pour développer les compétences langagières en français. Québec, QC : Université Laval.

Chi, A. M. L. (2003). An empirical study of the efficacy of integrating the teaching of dictionary use into a tertiary English curriculum in Hong Kong. Repéré à http://repository.ust.hk/ir/Record/1783.1-1058

Covington, M. V. (2000). Goal theory, motivation, and school achievement: An integrative review. Annual Review of Psychology, 51,171-200. http://dx.doi.org/10.1146/annurev.psych.51.1.171

Denham, S. A. (1998). Emotional development in young children. New York, NY : Guilford.

Duchesne, S., Vitaro, F., Larose, S. et Tremblay, R. E. (2008). Trajectories of anxiety during elementary-school years and the prediction of high school noncompletion. Journal of Youth and Adolescence, 37(9), 1134-1146. http://dx.doi.org/10.1007/s10964-007-9224-0

Eccles, J. S. et Wigfield, A. (2002). Motivational beliefs, values, and goals. Annual Review of Psychology, 53, 109-132. http://dx.doi.org/10.1146/annurev.psych.53.100901.135153

Field, A. (2013). Discovering statistics using IBM SPSS statistics (4éd.). Los Angeles, CA : Sage.

Fraser, C. A. (1999). The role of consulting a dictionary in reading and vocabulary learning. Canadian Journal of Applied Linguistics, 2(1-2), 73-89. Repéré à https://journals.lib.unb.ca/index.php/CJAL/article/view/19817

Fuertes-Olivera, P. A. et Tarp, S. (2011). Lexicography for the third millennium: Cognitive-oriented specialised dictionaries for learners. Iberica, 21, 141-161. Repéré à http://www.redalyc.org/articulo.oa?id=287023883008

Gagné, M. et Deci, E. L. (2009). Self-determination theory and work motivation. Journal of Organizational Behavior, 26(4), 331-362. http://dx.doi.org/10.1002/job.322

Gavriilidou, Z. (2013). Development and validation of the Strategy inventory for dictionary use (S. I. D. U.). International Journal of Lexicography, 26(2), 135-153. http://dx.doi.org/10.1093/ijl/ect007

Geay, B. (1995). Entre symbole et instrument, le dictionnaire à l'école primaire. Revue française de pédagogie, 113(1), 59-68. http://dx.doi.org/10.3406/rfp.1995.1218

Giasson, J. (2011). La lecture. Apprentissages et difficultés. Boucherville, QC : Gaëtan Morin.

Holzberger, D., Philipp, A. et Kunter, M. (2013). How teachers' self-efficacy is related to instructional quality: A longitudinal analysis. Journal of Educational Psychology, 105(3), 774-786. http://dx.doi.org/10.1037/a0032198

Karsenti, T. et Collin, S. (2013). Avantages et défis inhérents à l'usage des ordinateurs portables au primaire et au secondaire. Éducation et francophonie, 41(1), 94-122. http://dx.doi.org/10.7202/1015061ar

Laufer, B. (2000). Electronic dictionaries and incidental vocabulary acquisition: does technology make a difference? Dans U. Heid (dir.), EURALEX (p. 849-854). Repéré à http://euralex.org/wp-content/themes/euralex/proceedings/ Euralex\%202000/100 Batia\%20LAUFER Electronic\%20dictionaries\%20and\%20incidental\%20vocabulary\%20acqu isition does $\% 20$ technology $\% 20$ make $\% 20 a \% 20$ difference.pdf

Lew, R. (2013). From paper to electronic dictionaries: Evolving dictionary skills. Dans D. A. Kwary, N. Wulan et L. Musyahda (dir.), Lexicography and dictionaries in the information age. Selected papers from the $8^{\text {th }} A S I A L E X$ International Conference (p. 79-84). Repéré à https://repozytorium.amu.edu.pl/bitstream/10593/8140/1/Lew 2013\% 20From\%20paper\%20to\%20electronic\%20dictionaries.pdf

Linnenbrink-Garcia, L., Tyson, D. F. et Patall, E. A. (2008). When are achievement goal orientations beneficial for academic achievement? A closer look at main effects and moderating factors. International Review of Social Psychology, 21(1), 19-70. Repéré à https://www.cairn.info/revue-internationale-de-psychologie-sociale-2008-1-page-19.htm 
Ministère de l'Éducation, du Loisir et du Sport (MELS). (2011a). Progression des apprentissages au primaire. Français, langue d'enseignement. Repéré à http://www.education.gouv.qc.ca/fileadmin/site web/documents/education/jeunes/pfeq/ PDA PFEQ francais-langue-enseignement-primaire 2011.pdf

Ministère de l'Éducation, du Loisir et du Sport (MELS). (2011b). Progression des apprentissages au secondaire. Français, langue d'enseignement. Repéré à http://www.education.gouv.qc.ca/fileadmin/site web/documents/education/jeunes/ pfeq/PDA PFEQ francais-langue-enseignement-secondaire 2011.pdf

Moè, A., Pazzaglia, F. et Ronconi, L. (2010). When being able is not enough: the combined value of positive affect and self-efficacy for job satisfaction in teaching. Teaching and Teacher Education, 26(5), 1145-1153. http://dx.doi.org/10.1016/j.tate.2010.02.010

Nesi, H. (1999). The specification of dictionary reference skills in higher education. Dans R. R. K. Hartmann (dir.), Dictionaries in language learning. Recommendations, national reports and thematic reports from the TNP sub-project 9: Dictionaries (p. 53-67). Berlin : Frei Universität.

Neubach, A. et Cohen, A. D. (1988). Processing strategies and problems encountered in the use of dictionaries. Dictionaries: Journal of the Dictionary Society of North America, (10), 1-20. http://dx.doi.org/10.1353/dic.1988.0018

Pekrun, R., Elliot, A. J. et Maier, M. A. (2009). Achievement goals and achievement emotions: Testing a model of their joint relations with academic performance. Journal of Educational Psychology, 10(1), 115-135. http://dx.doi.org/10.1037/a0013383

Scholfield, P. (1982). Using the English dictionary for comprehension. TESOL Quarterly, 16(2), 185-194. http://dx.doi.org/10.2307/3586791

Scholfield, P. (1999). Dictionary use in reception. International Journal of Lexicography, 12(1), 13-34. http://dx.doi.org/10.1093/ijl/12.1.13

Scott, J. A., Nagy, W. E. et Flinspach, S. L. (2008). More than merely words: Redefining vocabulary learning in a culturally and linguistically diverse society. Dans A. E. Farstrup et S. J. Samuels (dir.), What research has to say about vocabulary instruction (p. 182-210). Newark : International Reading Association.

Stockless, A. et Beaupré, J. (2014). La compétence TIC chez les enseignants du primaire et du secondaire : rapport du sondage technopédagogique. Repéré à http://blogues.csaffluents.qc.ca/recit/files/2014/12/2014 1209 Rapport technopedagogique Final.pdf.

Tremblay, O. (2017). Le dictionnaire, un remède contre le syndrome de la page blanche. Correspondance, 22(9). Repéré à http://correspo.ccdmd.qc.ca/index.php/document/le-dictionnaire-un-remede-contre-le-syndrome-de-la-page-blanche

Vallerand, R. J. (dir.). (2006). Les fondements de la psychologie sociale (2éd.). Montréal, QC : Gaëtan Morin.

Villeneuve, S., Karsenti, T., Raby, C. et Meunier, H. (2012). Les futurs enseignants du Québec sont-ils technocompétents? Une analyse de la compétence professionnelle à intégrer les TIC. Revue internationale des technologies en pédagogie universitaire, 9(1-2), 78-99. http://dx.doi.org/10.7202/1012904ar

\section{Notes}

1 Tiré du site « Ouvrir le dictionnaire » du Centre collégial de développement de matériel didactique (CCDMD) : https://www.ccdmd.qc.ca/fr/modules/dictionnaire.

2 Il est à noter que près du tiers des enseignants de l'échantillon n'ont pu être considérés dans les analyses précédemment décrites $(n=191)$, car ils ne détenaient pas de dictionnaire électronique. Par conséquent, une analyse supplémentaire a été menée spécifiquement pour l'utilisation du dictionnaire papier $(n=292)$ pour vérifier si les différences relatives au niveau scolaire étaient similaires. Les résultats révèlent que l'effet du niveau scolaire est également significatif, ce qui confirme les résultats précédents. 
3 À nouveau, près du tiers des enseignants de l'échantillon n’ont pu être considérés dans les analyses décrites $(n=191)$, car ils ne détenaient pas de dictionnaire électronique. Par conséquent, une analyse supplémentaire a été réalisée spécifiquement pour vérifier la similarité des résultats obtenus relatifs au dictionnaire papier selon le niveau d'enseignement pour l'ensemble de l'échantillon détenant un dictionnaire papier $(n=292)$. Conformément aux résultats précédents, les analyses montrent une différence significative entre les buts usuels et enrichis, qui ne varient pas selon le niveau scolaire.

\section{Pour citer cet article}

Tremblay, O., Plante, I. et Fréchette-Simard, C. (2018). Les enseignants et le dictionnaire : sentiments, attitudes motivationnelles, connaissances déclarées et pratiques personnelles d'utilisation. Formation et profession, 26(3) 57-80. http://dx.doi.org/10.18162/fp.2018.452 